Editorial

\title{
Acknowlegement to Reviewers of OBM Geriatrics in 2020
}

OBM Geriatrics Editorial Office

LIDSEN Publishing Inc., 2000 Auburn Drive, One Chagrin Highlands, Suite 200, Beachwood, OH, USA; E-Mail: geriatrics@lidsen.com

\section{OBM Geriatrics}

2021, volume 5, issue 1

doi:10.21926/obm.geriatr.2101149
Received: January 04, 2021

Accepted: January 04, 2021

Published: January 06, 2021

\begin{abstract}
The editors of $O B M$ Geriatrics would like to express their sincere gratitude to the following reviewers for assessing manuscripts in 2020. We greatly appreciate the contribution of expert reviewers, which is crucial to the journal's editorial process. We aim to recognize reviewer contributions through several mechanisms, of which the annual publication of reviewer names is one. Reviewers receive a voucher entitling them to a discount on their next LIDSEN publication and can download a certificate of recognition directly from our submission system. Additionally, reviewers can sign up to the service Publons (https://publons.com) to receive recognition. Of course, in these initiatives we are careful not to compromise reviewer confidentiality. Many reviewers see their work as a voluntary and often unseen part of their role as researchers. We are grateful to the time reviewers donate to our journals and the contribution they make.
\end{abstract}

The editors of $O B M$ Geriatrics would like to express their sincere gratitude to the following reviewers for assessing manuscripts in 2020.

We greatly appreciate the contribution of expert reviewers, which is crucial to the journal's editorial process. We aim to recognize reviewer contributions through several mechanisms, of which the annual publication of reviewer names is one. Reviewers receive a voucher entitling them to a discount on their next LIDSEN publication and can download a certificate of recognition directly from our submission system. Additionally, reviewers can sign up to the service Publons (https://publons.com) to receive recognition. Of course, in these initiatives we are careful not to

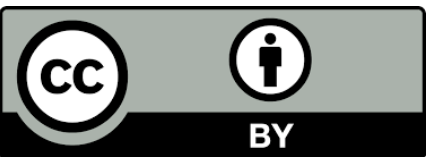

(C) 2021 by the author. This is an open access article distributed under the conditions of the Creative Commons by Attribution License, which permits unrestricted use, distribution, and reproduction in any medium or format, provided the original work is correctly cited. 
compromise reviewer confidentiality. Many reviewers see their work as a voluntary and often unseen part of their role as researchers. We are grateful to the time reviewers donate to our journals and the contribution they make.

If you are interested in becoming a reviewer for OBM Geriatrics, see the link at the bottom of the webpage http://lidsen.com/joinus.

The following reviewed for OBM Geriatrics in 2020:

\begin{tabular}{|c|c|c|}
\hline Alan J. Sinclair & Alessandro Leparulo & Alex J. Bishop \\
\hline Ana Raquel Ortega & Anna K. Koch & Annelien A. Duits \\
\hline Antonina Luca & Arthur Yin Fan & Ashley E. Ermer \\
\hline Ashley Herda & Ashwini Namasivayam-MacDonald & Audun Osland Vik-Mo \\
\hline Azucena Guzman & Balaji Krishnan & Bård Bogen \\
\hline Byron Creese & Catherine G. Ambrose & Catherine M. Headley \\
\hline Catherine Rogers-Clark & Cathy Treadaway & Chariklia Tziraki \\
\hline Cinda L. Hugos & Clara Bonanad & Daniel R. George \\
\hline Devin Wahl & Dimity Pond & Domenico De Berardis \\
\hline Donatella R. Petretto & Douglas G. Walker & Eleonora Mess \\
\hline Elisa Ogawa & Elizabeth A. Huntoon & Elizabeth M. Goldberg \\
\hline Elizabeth M. Orsega-Smith & Elliot Murphy & Emerson Sebastião \\
\hline Emmanuelle Cugy & Emmeline I. Ayers & Estibaliz Capetillo-Zarate \\
\hline Feng-Hua Ellen Loh & Fiona E.A. McGuigan & Foteini Christidi \\
\hline Giovanni Falsini & Gisela E. Hagberg & Haruka Tohara \\
\hline Helen Lavretsky & Hidehiro Ishikawa & Hui-Ju Young \\
\hline Ioannis Paliokas & Ivan Julian & Jaime A. Garcia \\
\hline James B. Robinson & James D Adams & Jennifer Perion \\
\hline Jeries Nashashibi & Jessica Kelliher Rabon & Jiraporn Sri-On \\
\hline João Pinho & Jorge Llibre Guerra & José María Pego-Reigosa \\
\hline Jose Losa-Reyna & Juan Fortea & Julie M. Nightingale \\
\hline Karen Harrison Dening & Katarzyna Kotfis & Kathlyn J. Ronaldson \\
\hline Kerry Jones & Khalid Iqbal & Koji Naruishi \\
\hline Kunakorn Atchaneeyasakul & Kyle Allen & Ladislav Volicer \\
\hline Laura Booi & Laura Cubero-Plazas & Laura E. Jonkman \\
\hline Laura Piccio & Laurens JL De Cocker & Lesley Ward \\
\hline Lisa Reider & Luca Cravello & Mamta Sapra \\
\hline Marco Sardiello & Maria Ehn & Mark S. Kindy \\
\hline Marta Monzón & Martin Hemels & Mary L Bouxsein \\
\hline
\end{tabular}




\begin{tabular}{|l|l|l|}
\hline Mary V Seeman & Masayoshi Kawakami & Melanie J. Chandler \\
\hline Melanie Handley & Michael Fossel & Mikhail Inyushin \\
\hline Mohit Kothari & Namkee G Choi & Natalie S. Ryan \\
\hline Nicholas Pound & Nicola Vanacore & Noriko Tanaka \\
\hline P. Hemachandra Reddy & Pedro Castro & Pedro Modrego \\
\hline Pere Clavé & Peter Hogg & Priyanka Shrestha \\
\hline R. M. Damian Holsinger & Rebecca B. Porter & Roy G. Beran \\
\hline Sara LaHue & Sarah A. Arias & Serge Gauthier \\
\hline Shinji Teramoto & Shuping Xiong & Simarjit Singhrao \\
\hline Sneha Mantri & Stefanie. Schreiber & Stephen D. Anton \\
\hline Susan E Mandel & Susan Freter & Susanne Finnegan \\
\hline T. Snijders & Takayuki Watabe & Tamara Backhouse \\
\hline Tamas Fulop & Tara Brennan-Speranza & Terry Fulmer \\
\hline Teruhiko Koike & Tharshanah Thayabaranathan & Tracy Wharton \\
\hline U. Anyanwagu & Vanessa A.D. Wilson & Vicki Winstead \\
\hline Victor Fu & Vincenzo Cimino & Viral N. Shah \\
\hline Xudong Huang & Yanjiao Zhou & Ye-Fong Du \\
\hline Yin Wu & & \\
\hline
\end{tabular}

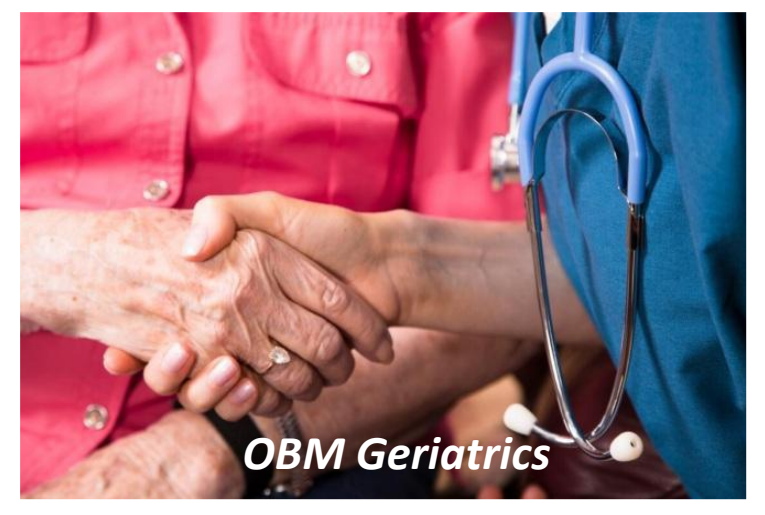

Enjoy $O B M$ Geriatrics by:

1. Submitting a manuscript

2. Joining in volunteer reviewer bank

3. Joining Editorial Board

4. Guest editing a special issue

For more details, please visit:

http://www.lidsen.com/journals/geriatrics 